CERN-TH/2000-219, hep-th/0007173

\title{
From quiver diagrams to particle physics
}

\author{
Angel M. Uranga \\ Theory Division, CERN \\ CH-1211 Geneva 23, Switzerland \\ Angel.Uranga@cern.ch
}

\begin{abstract}
Recent scenarios of phenomenologically realistic string compactifications involve the existence of gauge sectors localized on D-branes at singular points of Calabi-Yau threefolds. The spectrum and interactions in these gauge sectors are determined by the local geometry of the singularity, and can be encoded in quiver diagrams. We discuss the physical models arising for the simplest case of orbifold singularities, and generalize to non-orbifold singularities and orientifold singularities. Finally we show that relatively simple singularities lead to gauge sectors surprisingly close to the standard model of elementary particles.
\end{abstract}

\footnotetext{
${ }^{1}$ Invited lecture delivered at the Third European Congress of Mathematics, Barcelona, Spain, 10-14 July, 2000
} 


\section{Introduction}

The construction of string theory models which at low energies reproduce the basic features of the standard model of elementary particles (or some extension thereof) is a non-trivial task from the physical point of view. Interestingly, it also involves beautiful mathematics, and leads to a rich interplay between the physical and mathematical points of view. To quote a traditional example, the construction of four-dimensional $\mathcal{N}=1$ supersymmetric heterotic string vacua [1] involves the study of stable $G$-bundles on CalabiYau threefolds, with $G$ a subgroup of $E_{8} \times E_{8}$ or $S O(32)$ (for recent results on this approach, see [2]).

Recent developments in string theory have led to new kinds of string theory vacua with potential phenomenological interest. A particular class [4] corresponds to compactification on a Calabi-Yau threefold $X$ with gauge bundles defined on subvarieties of the internal space (times non-compact spacetime). In physical terms, the compactification includes a set of D-branes [3], which are dynamical extended objects partially wrapped on the internal manifold, and filling non-compact spacetime. Their dynamics is controlled by a gauge theory defined on their world-volume. The rank of the gauge bundle they carry is usually referred to as the number of D-branes. In the following we center on the simplest case of D3-branes in type IIB string theory, where each D-brane spans four-dimensional spacetime times a point $P \in X$. The properties of the corresponding gauge theory sector in the low-energy theory are then determined in terms of the local geometry of $X$ around $P$.

For a set of D3-branes sitting at a smooth point in $X$, the low-energy gauge field theory on the world-volume has $\mathcal{N}=4$ supersymmetry, and is therefore non-chiral and phenomenologically uninteresting. Chiral gauge sectors arise when D3-branes sit at singular points in $X$. Singularities preserving at least $\mathcal{N}=1$ supersymmetry, and at a finite distance in Calabi-Yau moduli space must be Gorenstein canonical singularities.

In Section 2 we discuss several systems of branes at singular points in non-compact Calabi-Yau spaces. In section 2.1 we center on the simple case of threefold quotient singularities, which leads to an implementation of the McKay correspondence in string theory. In section 2.2. and 2.3 we point out several generalizations suggested by string theory. In section 3 we discuss how an extremely simple $\mathbf{Z}_{\mathbf{3}}$ quotient singularity leads to gauge groups and particle contents remarkably similar to those of the (minimal supersymmetric) standard model. 
I am grateful to G. Aldazabal, A. Hanany, L. E. Ibáñez, J. Park, F. Quevedo and R. Rabadán for collaboration and useful discussion on these issues. I also thank M. González for encouragement and support.

\section{Branes at singularities}

\subsection{Branes at orbifold singularities}

Let $\Gamma$ be a discrete group of $S U(3)$, and $\left\{\mathbf{r}_{i}\right\}$ the set of its unitary irreducible representations. We want to consider a set of D3-branes at the origin of $\mathbf{C}^{\mathbf{3}} / \Gamma$, where $\Gamma$ acts on $\mathbf{C}^{3}$ through a three-dimensional representation $\mathcal{R}_{\mathbf{3}}$.

Consider first a set of $N$ D3-branes at the origin in $\mathbf{C}^{3}$, labeled by an index $a=1, \ldots, N$ referred to as Chan-Paton index. Quantization of open strings with endpoints of the D3-branes leads to a set of dynamical fields propagating on the D3-brane world-volume. In terms of $\mathcal{N}=1$ supersymmetry multiplets, the corresponding gauge field theory contains a set of vector multiplets (each containing one gauge field and one complex fermion) with gauge group $U(N)$, and three chiral multiplets $\Phi^{a}, a=1,2,3$ (each containing one complex scalar and one complex fermion). The latter transform in the adjoint representation of $U(N)$ and form a triplet under the $S U(3)$ action on $\mathbf{C}^{\mathbf{3}}$. The interactions are encoded in the superpotential function $W=\epsilon_{a b c} \operatorname{tr}\left(\Phi^{a} \Phi^{b} \Phi^{c}\right)$, where $\epsilon_{a b c}$ correspond to the components of the $S U(3)$ invariant tensor.

Following [5] (see [6, 7] for generalizations, and 8, 9, 10, 11] for related discussions) the field theory on D3-branes at the origin in $\mathbf{C}^{3} / \Gamma$ is obtained from the above field theory associated to $N$ D3-branes in flat space, by keeping the states which are invariant under the combined action of $\Gamma$ on $\mathbf{C}^{3}$ (as determined by $\mathcal{R}_{\mathbf{3}}$ ) and on the space of Chan-Paton indices (through a $N$ dimensional representation $\mathcal{R}$, with decomposition $\left.\mathcal{R}=\bigoplus_{i} N_{i} \mathbf{r}_{i}\right)$. Following [9], we regard fields in the adjoint of $U(N)$ as $\operatorname{Hom}\left(\mathbf{C}^{N}, \mathbf{C}^{N}\right)$. The projection on the $\mathcal{N}=1$ vector multiplets leaves the following fields

$$
\operatorname{Hom}\left(\mathbf{C}^{N}, \mathbf{C}^{N}\right)^{\Gamma}=\bigoplus_{i} \operatorname{Hom}\left(\mathbf{C}^{N_{i}}, \mathbf{C}^{N_{i}}\right)
$$

corresponding to a gauge group $\prod_{i} U\left(N_{i}\right)$. The projection on the $S U(3)$ triplet of $\mathcal{N}=1$ chiral supermultiplets leaves the following fields

$$
\left(\mathcal{R}_{\mathbf{3}} \otimes \operatorname{Hom}\left(\mathbf{C}^{N}, \mathbf{C}^{N}\right)\right)^{\Gamma}=\bigoplus_{i, j} a_{i j}^{\mathbf{3}} \operatorname{Hom}\left(\mathbf{C}^{N_{i}}, \mathbf{C}^{N_{j}}\right)
$$


where $a_{i j}^{\mathbf{3}}$ are defined by $\mathcal{R}_{\mathbf{3}} \otimes \mathbf{r}_{i}=\bigoplus_{j} a_{i j}^{\mathbf{3}} \mathbf{r}_{j}$. Hence we obtain $a_{i j}^{\mathbf{3}} \mathcal{N}=1$ chiral multiplets transforming in the representation $\left(N_{i}, \bar{N}_{j}\right)$ of the gauge group. The superpotential is obtained by restricting the above one to the surviving fields.

The field content on the D3-brane world-volume can be encoded in a quiver diagram, where the $i^{t h}$ node represents the $U\left(N_{i}\right)$ factor in the gauge group, and $a_{i j}^{\mathbf{3}}$ oriented arrows from the $i^{\text {th }}$ to the $j^{\text {th }}$ node correspond to the $\mathcal{N}=1$ chiral multiplets in the $\left(N_{i}, \bar{N}_{j}\right)$ representation. Finally, closed triangles of oriented arrows are associated to superpotential couplings of the corresponding chiral multiplets. The quiver for a $\mathbf{C}^{\mathbf{3}} / \mathbf{Z}_{\mathbf{5}}$ singularity is depicted in Fig. 2a.

Several interesting mathematical connections arise at this point. For instance, the quiver diagrams encoding the field theory content and interactions coincide with the McKay quivers associated to the singularity, and which are related to the homology of the resolved space by the McKay correspondence [12]. The correspondence arises in the string theory context since branes giving rise to a specific gauge factor have the geometrical interpretation of higher-dimensional branes wrapped on homology cycles of the space (see [13] for a detailed description). Hence, gauge theory data (the quiver diagram) are related to the homology of the ambient space.

Also, if $\mathcal{R}$ is chosen to be the regular representation of $\Gamma$, the moduli space of vacua of the gauge theory corresponds to the space of possible locations of the D3-brane, which is isomorphic to the transverse space $\mathbf{C}^{3} / \Gamma$. The construction of the moduli space amounts to performing a symplectic quotient in the subspace of fields subject to relations $\frac{\partial W}{\partial \Phi_{i}}=0$ (F-term constraints) determined by the superpotential [7]. It provides the string theory counterpart of the construction of $\mathbf{C}^{\mathbf{3}} / \boldsymbol{\Gamma}$ as the moduli of representations of a quiver diagram with relations 14. In the particular case $\gamma \subset S U(2)$, studied in [5, 6], one recovers the hyperkähler quotient construction of ALE spaces [15]. String theory also provides a description of the resolved spaces, by a suitable modification of the symplectic quotient due to a non-zero moment map (D-term).

We conclude this section with some physical considerations. In string theory D-branes are sources of certain $p$-form gauge fields from the closed string sector, whose equations of motion may impose certain consistency conditions on the D-brane configuration. In the particular case of D3-branes at $\mathbf{C}^{3} / \Gamma$ singularities, the equations of motion for fields located at the singularity im- 
pose the so-called twisted tadpole cancellation conditions, which for quotient singularities amount to the vanishing of the character of the representation $\mathcal{R}$. This constraint is equivalent to the cancellation of non-abelian anomalies in the gauge field theory on the D3-branes world-volume [16]. They also imply that the remaining mixed $U(1)$ - non-abelian anomalies have a factorized form and are cancelled by a version of the Green-Schwarz mechanism [17. The anomalous $U(1)$ factors become massive and disappear from the low-energy dynamics.

\subsection{Generalizations}

\subsubsection{Non-orbifold singularities}

There is no simple recipe to obtain the field theory on the world-volume of stacks of D3-branes at a general singularity. However, the requirement that its moduli space should correspond to the space of possible locations of Dbranes in the transverse space is enough to determine the field theory in the simple example of the conifold singularity $X_{\text {con }}$ [18], defined by the hypersurface $x^{2}+y^{2}+z^{2}+w^{2}=0$ in $\mathbf{C}^{4}$. A set of $N$ D3-branes at a conifold singularity yields a $\mathcal{N}=1$ supersymmetric field theory with gauge group $U(N) \times U(N)$ and chiral multiplets $A_{i}, B_{i}, i=1,2$ in the representations $(N, \bar{N})$ and $(\bar{N}, N)$ under the gauge group, and in the representations $(2,1)_{1 / 2},(1,2)_{1 / 2}$ under the $S U(2)^{2} \times U(1)$ symmetry group of $X_{c o n}$. The interactions are determined by a quartic superpotential $W=\epsilon^{i j} \epsilon^{k l} \operatorname{tr} A_{i} B_{j} A_{k} B_{l}$.

This example provides a whole new family of models [19] (see [20, 21] for a related discussion) corresponding to D3-branes at quotients of the conifold, $X_{\text {con }} / \Gamma$, with $\Gamma$ a subgroup of $S U(2) \times S U(2)$ in order to preserve $\mathcal{N}=1$ supersymmetry. The strategy is, as in section 2.1, to start with D3-branes at $X_{\text {con }}$, embed the action of $\Gamma$ on the Chan-Paton indices, and keep only fields which are invariant under the combined geometrical and Chan-Paton action of $\Gamma$. The resulting field theories can be encoded in a quiver diagram, with nodes and arrows corresponding to gauge factors and chiral multiplets, and superpotential terms correspond to closed polygons formed by four arrows. When $\Gamma$ acts on the Chan-Paton indices in the regular representation, the moduli space of the gauge theory is a symplectic quotient subject to F-term constraints from the superpotential, as obtained in explicit examples [22, 23], generalizing the orbifold result. The string theory construction also suggest these quiver diagrams also encode the homology of the resolution of $X_{\text {con }} / \Gamma$. 


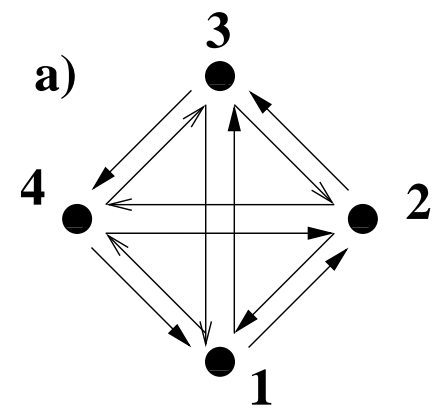

$\mathbf{x} \mathbf{y} \mathbf{z}=\mathbf{w}^{2}$ b)

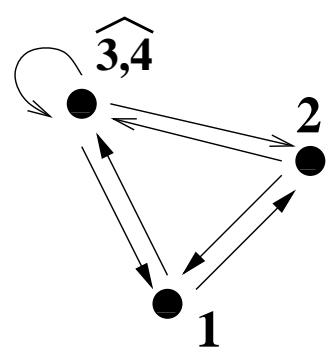

$\mathbf{x} \mathbf{y}=\mathbf{z} \mathbf{w}^{2}$ c)

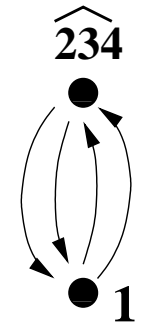

$\mathbf{x} \mathbf{y}=\mathbf{z} \mathbf{w}$

Figure 1: Figure a) shows the quiver diagram for a $\mathbf{C}^{\mathbf{3}} /\left(\mathbf{Z}_{\mathbf{2}} \times \mathbf{Z}_{\mathbf{2}}\right)$ singularity, which can be defined as the hypersurface $x y z=w^{2}$ in $\mathbf{C}^{4}$. Partial blow-ups resolve it to the suspended pinch point singularity $\left(x y=z w^{2}\right)$, and the conifold $(x y=z w)$, whose quiver diagrams are shown in Figures b) and c). Each blow-up is reflected on the quiver as the joining of two nodes (the joint node is denoted by a hat), and the disappearance of certain arrows (depicted with thin heads).

A general recipe to obtain the field theory on D3-branes at a general toric singularities $X$ was proposed in 24] (see [25] for a more general discussion). It is based on realizing $X$ as a partial resolution of a threefold quotient singularity $\mathbf{C}^{3} / \Gamma$ for suitable $\Gamma \subset S U(3)$. The construction requires a precise identification of the effect of the resolution on the field theory, which is a specific Higgs mechanism in which several gauge factors break to their diagonal subgroup, and some chiral multiplets become massive and disappear from the light spectrum. The detailed map has been worked out in some explicit examples, e.g. in [24, 26], and provides an explanation for the existence of quiver diagrams for non-orbifold singularities. They are obtained by joining nodes and deleting arrows in the quiver of the initial quotient singularity, as dictated by the Higgs mechanism in the field theory. The quiver version of the resolution of the $\mathbf{C}^{\mathbf{3}} /\left(\mathbf{Z}_{\mathbf{2}} \times \mathbf{Z}_{\mathbf{2}}\right)$ singularity to the conifold is shown in Figure 1 [24]. It would be interesting to obtain a more precise characterization of these operations on quiver diagrams.

Another interesting question is related to the uniqueness of the field theory corresponding to a given singularity [25]. In some cases, different field theories may have isomorphic moduli spaces. Mathematically, the representation moduli of the corresponding quivers with relations are isomorphic. 

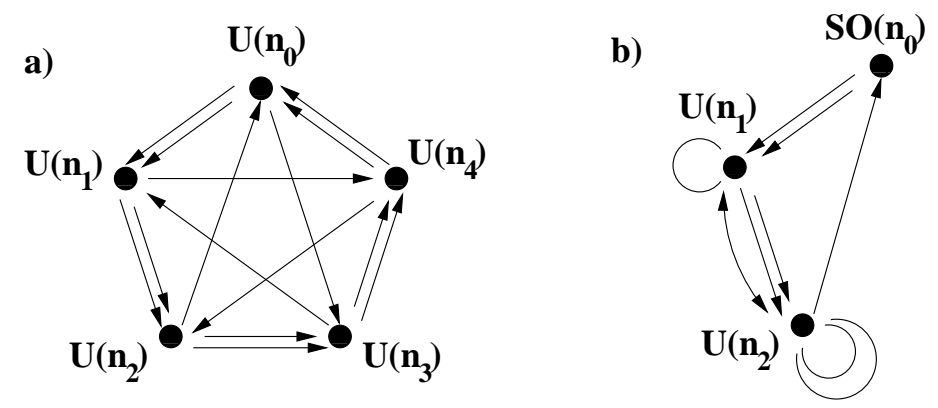

Figure 2: Figure a) shows the quiver diagram for the $\mathbf{C}^{\mathbf{3}} / \mathbf{Z}_{\mathbf{5}}$ singularity, with $\mathbf{Z}_{\mathbf{5}}$ action $\left(z_{1}, z_{2}, z_{3}\right) \rightarrow\left(e^{2 \pi i \frac{1}{5}} z_{1}, e^{2 \pi i \frac{1}{5}} z_{2}, e^{2 \pi i \frac{-2}{5}} z_{3}\right)$. Figure b) shows the quiver of the singularity after an orientifold projection.

From the physical point of view, the equality of moduli spaces may in some cases [19, 22] be a reflection of Seiberg duality, a non-trivial infrared equivalence of seemingly different field theories.

\subsubsection{Orientifold projections}

Type IIB string theory is invariant under an operation $\Omega$ which reverses the orientation on the world-sheet. Hence it is possible to consider modding out type IIB configurations by $\Omega$, possibly accompanied by a geometric involution $g$, also leaving the theory invariant [27, 28]. The new configurations thus obtained are called orientifolds, and are characterized by the inclusion of non-orientable world-sheets in the string theory perturbative expansion.

We are interested in studying D3-branes at orientifold threefold singularities, i.e. $\Omega g$ quotients of the system of D3-branes at singularities in CY threefolds. The best studied case is again that of quotient singularities $\mathbf{C}^{3} / \Gamma$, and for the sake of clarity here we center on $\Gamma=\mathbf{Z}_{\mathbf{N}}$ with the action of the generator $\theta$ of $\mathbf{Z}_{N}$ on $\mathbf{C}^{3}$ represented by $\gamma=\operatorname{diag}\left(e^{2 \pi i t_{1} / N}, e^{2 \pi i t_{2} / N}, e^{2 \pi i t_{3} / N}\right)$, with $t_{i}$ integers defined modulo $N$, and $\sum_{a=1}^{3} t_{a}=0 \bmod N$. Let us embed the action of $\theta$ on the Chan-Paton indices by a diagonal matrix $\gamma_{\theta, 3}$ with $N_{k}$ entries $e^{2 \pi i k / N}$. The field theory one obtains has vector multiplets with gauge group $\prod_{i=1}^{N} U\left(N_{i}\right)$, and chiral multiplets $\Phi_{i, i+t_{a}}^{a}, a=1, \ldots, 3, i=1, \ldots, N$ in the representation $\left(N_{i}, \bar{N}_{i+t_{a}}\right)$.

The orientifold action may also be embedded in the space of Chan-Paton indices, through a matrix $\gamma_{\Omega g, 3}$. The representations of $\Gamma$ and $\Omega g$ are usually 
constrained from mutual consistency requirements [27, 28]. Several solutions to these conditions are known (quite exhaustively in the two-fold case [29]), but a complete classification is lacking. In the following we center on a concrete example [17] where $g$ inverts all coordinates in $\mathbf{C}^{3}$, and exchanges closed string fields in oppositely twisted sectors, i.e. has a non-trivial action on the homology cycles shrunk at the singularity. We also choose $\gamma_{\Omega g, 3}$ symmetric and such that exchanges the eigenspaces of conjugate eigenvalues in $\gamma_{\theta, 3}$. The action of $\mathbf{Z}_{N}$ on Chan-Paton indices is therefore constrained to form a real representation, $N_{k}=N_{-k}$.

The effect of the orientifold projection on the spectrum is as follows. Gauge factors associated to conjugate irreducible representations of $\mathbf{Z}_{N}$ are identified, and unitary gauge factors associated to real representations are reduced to their maximal orthogonal subgroups. Correspondingly, the chiral multiplets $\Phi_{i, i+t_{a}}^{a}$ and $\Phi_{-i-t_{a},-i}^{a}$ are identified. When $i+t_{a}=-i$ the bifundamental field is projected down to a two-index antisymmetric representation of the unitary gauge factor after the projection. The quiver diagram for an orientifold of a $\mathbf{C}^{\mathbf{3}} / \mathbf{Z}_{\mathbf{5}}$ singularity is depicted in Figure $2 \mathrm{~b}$.

The effect on the quiver diagram of $\mathbf{C}^{3} / \Gamma$ is an identification of nodes and arrows related by a $\mathbf{Z}_{2}$ action, with a specific prescription for nodes and arrows which are mapped to themselves (it would be interesting to characterize these mappings more precisely in order to allow the classification of resulting models). One may wonder about the meaning of the resulting quiver. String theory suggests it encodes the information about the homology of the resolved orientifold singularity. In fact, the string theory counting of homology cycles in the resolved space (by counting of twisted sector blow-up moduli) gives roughly speaking half the number encountered before the orientifold projection, due to the non-trivial action of $g$ by exchanging oppositely twisted sectors. This agrees with the counting of nodes in the orientifolded quiver, which also gives half the number encountered before the orientifold projection.

We conclude by pointing out that orientifolds of non-orbifold singularities can be constructed as partial resolutions of orientifold of quotient singularities. Preliminary results on some simple examples [30] indicate that the effect of the orientifold projection on the quiver of the non-orbifold singularity is also a $\mathbf{Z}_{\mathbf{2}}$ involution, and that the orientifold singularity can be constructed as a symplectic quotient on the space of fields constrained by the F-terms conditions. 


\section{Particle physics}

In this section we discuss particular examples of singularities leading to interesting low-energy field theories, in that they resemble the structure of the (minimal supersymmetric) standard model (or some extension thereof) which we now review. Such models would provide phenomenologically interesting string theory vacua when embedded in a compact Calabi-Yau context.

\subsection{Review of the (minimal supersymmetric) standard model}

All known non-gravitational interactions between elementary particles are described by the quantum field theory known as standard model. The simplest supersymmetric extension of this model contains vector multiplets with gauge group $S U(3) \times S U(2) \times U(1)$. It also contains a set of chiral multiplets transforming in three copies of the representation

$$
(3,2)_{1 / 6}+(\overline{3}, 1)_{-2 / 3}+(\overline{3}, 1)_{1 / 3}+(1,2)_{1 / 2}+(1,1)_{1},
$$

where subscripts denote $U(1)$ charges. Successful breaking of the electroweak interactions requires also at least one chiral multiplet in the representation $(1,2)_{1 / 2}+(1,2)_{-1 / 2}$.

Interactions are encoded in a set of complicated gauge invariant functions of these chiral multiplets, the superpotential and gauge kinetic functions, which are holomorphic, and the Kähler potential, which is real. We will skip their details since realistic phenomenology usually involves additional model-dependent assumptions, like additional global symmetries.

\subsection{Realistic models from $\mathrm{Z}_{3}$ singularities}

The replication of families is an intriguing feature of the above field theory. To reproduce it from branes at singularities, the case of $\mathbf{C}^{3} / \mathbf{Z}_{3}$ with the $\mathbf{Z}_{3}$ action defined by $\left(z_{1}, z_{2}, z_{3}\right) \rightarrow\left(e^{2 \pi i / 3} z_{1}, e^{2 \pi i / 3 z_{2}}, e^{2 \pi i / 3} z_{3}\right)$, is singled out, in that it leads to natural triplication. The two examples we are to consider are based on this singularity.

The first example we consider forms a subsector of the model considered in [31]. It is constructed by placing eleven D3-branes on the ori-

entifold of the $\mathbf{C}^{3} / \mathbf{Z}_{3}$ singularity, introduced in section 2.2.2. We choose 
$\gamma_{\theta, 3}=\operatorname{diag}\left(1, e^{2 \pi i / 3} \mathbf{1}_{5}, e^{-2 \pi i / 3} \mathbf{1}_{5}\right)$. Following our rules above, we obtain vector multiplets with gauge group $S U(5)$ (the $U(1)$ factor disappears from the light spectrum as explained in section 2.1), and there are chiral multiplets in three copies of the representation $5+\overline{10}$. This spectrum resembles the structure of $S U(5)$ grand unified theories, which reproduce the spectrum of the minimal supersymmetric standard model when an additional field in the adjoint representation is present to break $S U(5)$ to the standard model group through the Higgs mechanism (an additional $5+\overline{5}$ pair is further required for electroweak symmetry breaking). Unfortunately, all Higgs fields are absent in our field theory, which therefore is suggestive but not truly realistic.

Our second example is based on the $\mathbf{C}^{3} / \mathbf{Z}_{3}$ orbifold (rather than orientifold) singularity $\mathbf{f}$, with Chan-Paton embedding $\gamma_{\theta, 3}=\operatorname{diag}\left(\mathbf{1}_{\mathbf{3}}, e^{2 \pi i / 3} \mathbf{1}_{2}\right.$, $\left.e^{-2 \pi i / 3}\right)$. This embedding does not satisfy the tadpole cancellation condition stated in section 2.1 (the character of the representation of $\mathbf{Z}_{3}$ is non-zero). The problem can be solved by introducing an additional set of D-branes, for instance $\mathrm{D} 77_{a}$-branes, with $a=1,2,3$, wrapped on the complex surfaces $z_{a}=0$, which do not break further supersymmetries. For non-compact spaces these additional branes are non-dynamical, but they contribute additional fields in the four-dimensional world-volume of the D3-branes, arising from open strings stretched between D3- and D7-branes. Hence the corresponding gauge field theories correspond to extended quivers, where additional nodes correspond to the global symmetries (symmetries on the D7-branes), and additional arrows correspond to 3-7 and 7-3 string states. These quiver diagrams generalize to the threefold case those in [5]. Their moduli spaces therefore provide a generalization of the Kronheimer-Nakajima construction of the moduli space of instantons on ALE spaces [34].

The $\mathbf{Z}_{3}$ acts on the space of D7-brane Chan-Paton indices, through matrices $\gamma_{\theta, 7_{a}}$. The modified tadpole cancellation conditions (which reduce to $\left.\sum_{a} \operatorname{tr} \gamma_{\theta, 7_{a}}+3 \operatorname{tr} \gamma_{\theta, 3}=0\right)$ are satisfied for the very symmetric choice $\operatorname{tr} \gamma_{\theta, 7_{a}}=-\operatorname{tr} \gamma_{\theta, 3}, a=1,2,3$. Choosing e.g. $\gamma_{\theta, 7_{a}}=\operatorname{diag}\left(e^{2 \pi i / 3}, e^{-2 \pi i / 3} \mathbf{1}_{2}\right)$ we obtain the following spectrum

$$
\begin{array}{ccc}
3-3 \text { strings } & \text { Gauge group } & S U(3) \times S U(2) \times U(1) \\
& \text { Chiral multiplets } & 3\left[(3,2)_{1 / 6}+(1,2)_{1 / 2}+(\overline{3}, 1)_{-2 / 3}\right] \\
3-7_{a} \text { strings } & \text { Chiral multiplets } & (3,1)_{-1 / 3}+2(1,2)_{-1 / 2}+ \\
a=1,2,3 & & +2(\overline{3}, 1)_{1 / 3}+(1,1)_{1}
\end{array}
$$

\footnotetext{
${ }^{2}$ This model has been studied in 32 and is similar to a subsector of models in [33].
} 


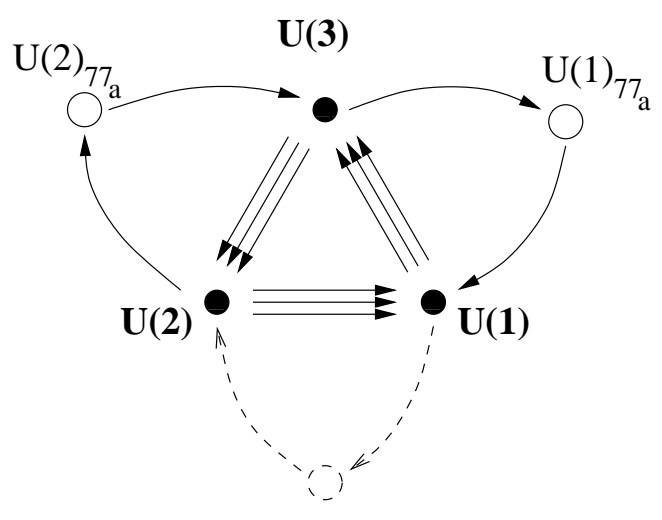

Figure 3: Quiver diagram of a system of D3- and D7-branes at a $\mathbf{C}^{\mathbf{3}} / \mathbf{Z}_{\mathbf{3}}$ singularity, reproducing a spectrum close to the (minimal supersymmetric) standard model. The 3-7 sector is triplicated due to the three kinds of D7-branes we have introduced. The dotted node and arrows are present in the generic quiver, but not for our specific choice of Chan-Paton representations. Notice that white nodes correspond to global symmetries of the D3-brane field theory, and that only one of the three $U(1)$ symmetries of the D3-branes survives at low energies.

We have included the charges under the only non-anomalous linear combinations of the $U(1)$ factors in the original $U(3) \times U(2) \times U(1)$ gauge group. This $U(1)$ does not become massive and plays the role of hypercharge in the standard model we have just constructed. The quiver diagram of this gauge theory is shown in Figure 3 .

Notice how close to the spectrum in section 3.1 one can get using very simple singularities. In particular, the later model differs from the MSSM just in that it produces three Higgs pairs instead of one, and in fact constitutes one of the simplest semi-realistic string models ever built. Since such constructions would provide a rationale for the existence of three generations (one per complex transverse dimensions), and for hypercharge assignments (see [35 for discussion of hypercharge in a other brane contexts), we believe these examples illustrate the phenomenological interest of string theory compactifications with branes at singularities.

\section{References}


[1] P. Candelas, G. T. Horowitz, A. Strominger, E. Witten, Nucl.Phys. B258(1985)46.

[2] R. Donagi, A. Lukas, B. A. Ovrut, D. Waldram, JHEP 9905(1999)018, hep-th/9811168;JHEP 9906(1999)034, hep-th/9901009, and references therein.

[3] J. Polchinski, hep-th/9611050.

[4] N. Arkani-Hamed, S. Dimopoulos, G. Dvali, Phys. Lett. B429 (1998) 263, hep-ph/9803315; I. Antoniadis, N. Arkani-Hamed, S. Dimopoulos, G. Dvali, Phys. Lett. B436 (1998) 257, hep-ph/9804398.

[5] M. R. Douglas, G. Moore, hep-th/9603167.

[6] C. V. Johnson, R. C. Myers, Phys. Rev. D55 (1997) 6382, hepth/9610140.

[7] M. R. Douglas, B. R. Greene, D. R. Morrison, Nucl. Phys. B506 (1997) 84, hep-th/9704151.

[8] S. Kachru, E. Silverstein, Phys. Rev. Lett. 80 (1998) 4855, hepth/9802183.

[9] A. Lawrence, N. Nekrasov, C. Vafa, Nucl. Phys. B533 (1998) 199, hepth/9803015.

[10] A. Hanany, A. M. Uranga, JHEP 9805(1998)013, hep-th/9805139.

[11] A. Hanany, Y.-H. He, JHEP 9902 (1999) 013, hep-th/9811183; B. R. Greene, C. I. Lazaroiu, M. Raugas, Nucl. Phys. B553 (1999) 711, hep-th/9811201; T. Muto, JHEP 9902 (1999) 008, hep-th/9811258.

[12] J. McKay, Proc. Symp. Pure Math. 37 (1980) 183; M. Reid, alggeom/9702016.

[13] D. E. Diaconescu, J. Gomis, hep-th/9906242.

[14] A. V. Sardo Infirri, alg-geom/9610005.

[15] P. B. Kronheimer, J. Diff. Geom. 29 (1989) 665.

[16] R. G. Leigh, M. Rozali, Phys. Rev. D59 (1999) 026004, hep-th/9807082.

[17] L. E. Ibáñez, R. Rabadán, A. M. Uranga, Nucl. Phys. B542 (1999) 112, hep-th/9808139. 
[18] I. R. Klebanov, E. Witten, Nucl. Phys. B536 (1998) 199, hepth/9807080.

[19] A. M. Uranga, JHEP 9901(1999)022, hep-th/9811004.

[20] K. Dasgupta, S. Mukhi, Nucl. Phys. B551 (1999) 204, hep-th/9811139.

[21] M. Aganagic, A. Karch, D. Lüst, A. Miemiec, Nucl.Phys. B569(2000)277, hep-th/9903093.

[22] R. von Unge, JHEP 9902 (1999) 023, hep-th/9901091.

[23] K. Oh, R. Tatar, JHEP 9910 (1999) 031, hep-th/9906012.

[24] D. R. Morrison, M. R. Plesser, Adv. Theor. Math. Phys. 3 (1999) 1, hep-th/9810201.

[25] B. Feng, A. Hanany, Y.-H. He, hep-th/0003085.

[26] C. Beasley, B. R. Greene, C. I. Lazaroiu, M. R. Plesser, Nucl.Phys. B566 (2000) 599, hep-th/9907186.

[27] A. Sagnotti in Cargese'87, in Non-perturbative quantum field theory, ed. G. Mack et al (Pergamon Press 88), pag. 521; G. Pradisi, A. Sagnotti, Phys. Lett. B216 (1989) 59; M. Bianchi, A. Sagnotti, Phys. Lett. B247(1990)517; Nucl.Phys. B361(1991)519.

[28] E. G. Gimon, J. Polchinski, Phys. Rev. D54 (1996) 1667, hepth/9601038; E. G. Gimon, C. V. Johnson, Nucl.Phys. B477 (1996) 715, hep-th/9604129; A. Dabholkar, J. Park, Nucl. Phys. B477 (1996) 701, hep-th/9604178.

[29] K. Intriligator, Nucl.Phys. B496(1997)177, hep-th/9702038; J. D. Blum, K. Intriligator, Nucl.Phys. B506(1997)223, hep-th/9705030; Nucl. Phys. B506 (1997) 199, hep-th/9705044;

J. Park, A. M. Uranga, Nucl. Phys. B542 (1999) 139, hep-th/9808161;

J. Park, R. Rabadán, A. M. Uranga, Nucl.Phys. B570(2000)3, hepth/9907074; A. M. Uranga, Nucl.Phys. B577(2000)73, hep-th/9910155.

[30] J. Park, R. Rabadán, A. M. Uranga, Nucl.Phys.B570(2000)38, hepth/9907086.

[31] J. Lykken, E. Poppitz, S. P. Trivedi, Nucl. Phys. B543 (1999) 105, hepth/9806080. 
[32] G. Aldazabal, L. E. Ibáñez, F. Quevedo, A. M. Uranga, hep-th/0005067.

[33] G. Aldazabal, L. E. Ibáñez, F. Quevedo, JHEP 0001(2000)031, hepth/9909172.

[34] P. B. Kronheimer, H. Nakajima, Math. Ann. 288(1990)263.

[35] N. D. Lambert, P. C. West, JHEP 9909(1999)021, hep-th/9908129;

I. Antoniadis, E. Kiritsis, T. N. Tomaras, hep-ph/0004214. 